\title{
Correction to: Acquisition of Tn6018-3' CS regions increases colistin MICs against Acinetobacter baumannii isolates harboring new variants of AbaRs
}

\author{
Mohammad Savari $^{1} \cdot$ Alireza Ekrami $^{2} \cdot$ Saeed Shoja $^{3}$ • Abbas Bahador ${ }^{1,4,5}$
}

Published online: 7 November 2017

(C) Institute of Microbiology, Academy of Sciences of the Czech Republic, v.v.i. 2017

\section{Correction to: Folia Microbiol}

$$
\text { https://doi.org/10.1007/s12223-017-0507-x }
$$

The published online version of this article contained a mistake. The correct affiliation of Alireza Ekrami should have been "Infectious and Tropical Diseases Research Center, Health Research Institute, Ahvaz Jundishapur University of Medical Sciences, Ahvaz, Iran". The authors regret this error.

The online version of the original article can be found at https://doi.org/ 10.1007/s12223-017-0507-x

Abbas Bahador

abahador@sina.tums.ac.ir; ab.bahador@gmail.com

1 Department of Microbiology, School of Medicine, Tehran University of Medical Sciences, Keshavarz Blvd, 100 Poursina Ave, Tehran 1416753955, Iran

2 Infectious and Tropical Disease Research Center, Health Research Institute, Ahvaz Jundishapur University of Medical Sciences, Ahvaz, Iran

3 Infectious and Tropical Disease Research Center, Hormozgan University of Medical Sciences, Bandar Abbas, Iran

4 Dental Research Center, Dentistry Research Institute, Tehran University of Medical Sciences, Tehran, Iran

5 Laser Research Center, Dentistry Research Institute, Tehran University of Medical Sciences, Tehran, Iran 